\title{
Anglicismos tempranos en el Perú del siglo XIX
}

\author{
Early anglicisms in the 19th Perú century
}

Carlos Arrizabalaga Lizárraga ${ }^{1}$

\begin{abstract}
RESUMEN
El artículo pone su atención sobre los anglicismos tempranos que se impusieron en muchos dialectos hispanoamericanos por el enorme peso del imperialismo comercial británico durante todo el siglo XIX y se hallan completamente adaptados a la fonética y a la morfología del castellano, y se transcriben con la ortografía castellana. Son palabras que, se diría, no parecen anglicismos, dado que están completamente asimiladas como "chompa" (de jumper"), "saco" y "chaqueta" (de jacket"), "lonche" (de "lunch"), "faite" (de "fighter"), "queque" (de "cake") y "gasfitero"(de" "gasfiter"). Se comenta el caso de lonche con detalle de su tratamiento lexicográfico. Además, se indica la presencia de fraseología procedente del inglés $y$, en sentido contrario, de algunos peruanismos en el inglés hablado en el sudoeste de Estados Unidos.
\end{abstract}

\section{Palabras clave}

Anglicismos, Perú, Siglo XIX, préstamo lingüístico, fraseología.

\begin{abstract}
The article focuses on the early Anglicisms imposed on many Hispanic dialects due to the great power of the British Commercial Imperialism all through the 19th century, which are completely adapted to the Spanish phonetics and morphology, and thus transcribed with the Spanish spelling. These are words that do not seem to be Anglicisms since they have been completely assimilated, such as "chompa" (from "jumper"), "saco" and "chaqueta" (from "jacket"), "lonche" (from "lunch"), "faite" (from "fighter"), "queque" (from "cake") and "gasfitero" (from "gasfiter"). The case of "lonche" is particularly mentioned regarding its lexicographical use. In addition, the article points out the existence of some phraseology taken from English, and also of some Peruvian expressions used in the English language spoken in the south west of the United States.
\end{abstract}

\section{Keywords}

Anglicisms, Peru, 19th century, loanword, phraseology.

Doctor en Filología Hispánica por la Universidad de Navarra (España). Ha sido profesor de la Universidad de Piura y profesor visitante en la Universidad Estatal de California en la Bahía de Monterrey. Ha publicado el estudio Gramaticalización en español americano. La perífrasis concomitante norperuana (2010) y también El dejo piurano y otros estudios (2008 y 2012), además de diversos artículos sobre dialectología, lexicología e historia de la lengua española ("Noticias de la desaparición del voseo en la costa norte del Perú" (2001); "Americanismos en la Descripción geográfica del partido de Piura de José Ignacio de Lecuanda (2007); "Comentarios a un pasaje de Gonzalo Fernández de Oviedo" (2007), "Imploraba que no lo maten. Usos del subjuntivo en español peruano (2009), "Ya con Felicitas fuimos a ver a buscar al párroco de Santa Catalina». Discordancia gramatical y gramaticalización en español peruano" (2011), "Barbarismos en las Papeletas lexicográficas de Ricardo Palma" (2003); "Garcilaso y la lexicografía peruana" (2009), y notas etimológicas sobre "Encalavernarse" y "Tracalada" (2010), y "Bamba" (2011). Actualmente, es editor de la revista Mercurio Peruano, en la Universidad de Piura. 
En Colombia y en toda Hispanoamérica - decía hace medio siglo el erudito colombiano Luis $\mathrm{F}$. Flórez- se emplean ahora muchas palabras del inglés norteamericano, cosa explicable por la expansión económica, política y militar de los Estados Unidos." Verdad que se notó entonces una profusión de anglicismos -para Jerónimo Malo más que profusión fue una "invasión" $y$, para muchos, innecesarios-, sobre todo en el habla coloquial de algunos, que ensartan sin parar expresiones contagiadas a través del cine y la música como "sunset", "show" (pronunciado "chou"), y otros muchos como "blazer", "fólder", "finepen" (también "pluma fuente", que es un calco), "percentil", "performance", "pioner", "point", "sachet", verbos como "resetear", "chequear", "chipear", "zipear", junto con colocaciones del tipo "tener feeling", "estar full", "haber chance", etc. Hay, aparte, invenciones graciosas como la composición (apoyada en la paronasia) que producen "cholywood" o "pichiroom". Aparte de la multitud de anglicismos en los deportes, muchos de los cuales son relativamente antiguos.

Muchos anglicismos se han instalado con gran firmeza y las empleamos con naturalidad, como el sargento Lituma que dice que va a "tirar una luqueada" con el revólver en la mano en Lituma en los Andes (Barcelona, Planeta, 1993: 61) pero otros anglicismos resultan huachafos como cuando Carreño, en la misma novela, quería bailar con la Meche cheak to cheak (221). ${ }^{2}$

Pero, quería llamar la atención sobre los anglicismos tempranos, los que llegaron a Sudamérica con los militares y comerciantes ingleses ya desde la época de la Independencia, $y$ se impusieron en muchos dialectos hispanoamericanos por el enorme peso del imperialismo comercial británico, que haría sentir su influencia hasta la primera Guerra Mundial. Me refiero a palabras que diríamos no parecen anglicismos, dado que están completamente asimiladas y adaptadas tanto fonética como ortográficamente (y morfológicamente también, claro) al castellano peruano. Podemos señalar al menos seis anglicismos tempranos: "chompa" (de "jumper"), "esplinático" (de "spleen", 'aburrimiento' o 'melancolía'), "faite" (de "fighter", por 'luchador'), "gasfitero" (de "gasfitter", fontanero), "guaipe" (de "wipe", "trapo'), "huacha" (de "washer", 'anilla') "lonche" (de "lunch"), "queque" (de "cake"), remarcable (de "remarkable") y "saco" (de "jacket", de donde viene también "chaqueta"), usuales en el Perú, además de los que se difundieron igualmente en otros países como "boicotear" y "reportero".

Martha Hildebrandt describió con esmerada pulcritud la introducción de "chompa" procedente del inglés británico a fines del siglo XIX, y la aparición de "faite" hacia 1880 (1994: 133-134). Podría hacerse la misma indagación con respecto a todos los demás vocablos, aunque no siempre es fácil encontrar fuentes que brinden testimonios tempranos de su introducción al español andino.

Los escritores suelen ser buenos observatorios del idioma, detectando con gran agudeza las innovaciones que se van produciendo. Algunas fueron ya señalados por Ricardo Palma en sus Papeletas lexicográficas (1903), aunque se mostrara algo reticente a los extranjerismos. $\mathrm{Su}$ hijo Clemente Palma incluía el vocablo chuzo (de "shoe"), en un glosario de argot limeño, apéndice de las Crónicas de su alter ego Apapucio Corrales (1938).

La influencia del inglés en el Perú fue muy temprana. Desde el momento mismo de la Independencia, los ingleses habían difundido sus modas y costumbres, el té y los casimires. En 1824, el acaudalado español Lucas de la Cotera abandonó Arequipa con las autoridades españolas, y en su lugar se instaló el cónsul inglés Passmore y 28 representantes de firmas comerciales británicas. Muy pronto, las mujeres elegantes de Arequipa se adornarían el cabello con bucles postizos importados de Albión bajo el nombre de "inglesas".

Inglaterra se adueña del comercio y arbitra las fronteras hasta provocar conflictos si sus intereses ven amenazados, como en caso del nitrato, componente principal de la pólvora. Entonces todo se pesaba en "libras" y se ponían

2 La frase inglesa correcta es cheek to cheek, es decir, "cachete con cachete". 
de moda los "bombines" (que subsisten en el Altiplano). También estuvieron a la moda los de castor de waterproof, según la festiva prosa de Segura (1968: 144).

Ricardo Palma registró la introducción de "boicotear" y "boicoteo", cuya historia es bien conocida: los clientes de un tendero irlandés de Londres, un tal Boycott, se negaron a seguir comprando en su tienda; esto fue en 1880, y dos décadas después, la palabra ya era bien conocida en Lima. Palma recoge estos préstamos a regañadientes, como en lunch: "Nadie prescinde ya de pronunciar o escribir la tal palabrita inglesa." Su comentario al respecto no puede ser más elocuente, solo cree aceptables "aquellos que se han impuesto ya en el lenguaje, y para los que no hay policía que alcance á expulsarlos de casa", sobre todo si resultan convenientes y no hay para ellos equivalentes en castellano. (1903: VIII). El propio Palma los utiliza en sus relatos y en sus cartas: "soy de los que creen que la democracia no está reñida con el confort" (1964: 1353).

Ricardo Alfaro en su Diccionario de anglicismos (1970) se mostraba implacable con el "barbarismo vulgar" propio de "gente zafia", y así calificaba a términos como "guachimán" y otros anglicismos del español americano, pero es que los españoles se equivocan al rechazar como "malsonantes" los anglicismos que son cotidianos para todos en los países americanos (1970:17). Muchos no verían la luz en el Diccionario oficial hasta después de muchas décadas.

En 1927, la Real Academia Española publica un Diccionario manual e ilustrado "con definiciones -decía Lapesa- más escuetas que el común y con multitud de vocablos y acepciones que están en uso, pero que por su origen extranjero, por su novedad no plenamente consolidada o por otras respetables consideraciones, no han tenido aún la aprobación de la Academia" (1996: 230). Julio Casares señaló que la obra nacía con vocación americanista (1950: 303). Confinados en ese lugar han quedado muchos americanismos procedentes del inglés, como "remarcable", "lonche" o "provisorio". Casares dijo que hubo "algunas inclusiones no bien justificadas" (1950: 303), pero lo que no parece justificado es que la Academia no incluyera estas y otras muchas palabras en el Diccionario oficial cuando por otra parte ella misma testificaba su uso continuado en las siguientes ediciones del Diccionario Manual (de 1950, 1983-5 y 1989).

En ese sentido, parece mentira que hayan tenido una historia tan diferente "lonche" y "ponche", cuando son tan parecidas. Ponche, forma hispanizada de punch, se aceptó enseguida por ser usual en ambas orillas del castellano y está desde 1880 en el Diccionario oficial, pero la palabra "lonche" (de "lunch"), solo recogida en el Diccionario Manual (1927), como propia de México, y con asterisco: recomendando que se reemplazara por "almuerzo". Y así estuvo décadas, como dice Manuel Seco, en una "cuarentena tácita", como muchos otros americanismos y extranjerismos que estuvieron "almacenados con sus corchetes en el Diccionario Manual en una especie de Limbo sin que la Academia terminase de decidir si las asimila oficialmente o les retiraba su interinidad" (Seco, 1993: 166).

Definitivamente en el caso de "lonche" su extensión y vitalidad merecía un mejor trato académico puesto que es de uso general y ha creado derivados plenamente aceptados como "lonchera", "lonchecito". Pues bien, ha tenido que ser esta última edición del Diccionario de la Academia, la 23. ${ }^{a}$ edición de 2014, la que ha incluido por fin "lonche", aunque entre las marcas geográficas no se incluye Perú ni Chile y la acepción 'comida ligera del mediodía' no es la usual en Sudamérica de comida ligera de la tarde, usualmente acompañada de té o cualquier bebida caliente. Incluye también "lonchera", con mejor fortuna, y "lonchería", establecimiento donde se ofrecen comidas ligeras, usual en México, Colombia y Venezuela.

Es posible establecer con cierta seguridad que se trata de un anglicismo temprano. $\mathrm{El}$ francés Paul Marcoy, al inicio de su viaje a través de América del Sur (hacia 1869), dice que se toma un "luncheon" (escrito en inglés del siglo XVI) con el cónsul inglés en Islay, y más adelante se toma también otro en casa del 
subprefecto de Urubamba, en el Cusco. Y la había mencionado ya el mismo Juan de Arona en su Diccionario de Peruanismos de 1882, al hablar de "las tabernas que bajo el epígrafe de Cerveza y Lunch" invadían Lima desde hacía unos diez años atrás" (1974: 175). José Gálvez recordaría luego que en Lima era costumbre el bizcochuelo, el buen chocolate y el vino dulce: "lo que con el progreso se trocó un día, gracias a Field, a los ingleses y a los tiempos en el famoso té con hojitas limeñas" (1965: 136) Del inglés decimonónico vienen también el adjetivo "remarcable", y el delicioso "queque", del que nos ofrece un testimonio temprano, en castellano, el alemán Heinrich Witt:

"La escasez de árboles nos llamó la atención como algo remarcable; nos dijeron que era parcialmente atribuible a la falta de agua, pero principalmente a la ociosidad de los habitantes que no querían darse el trabajo de plantarlos" (Witt, 1987: 27); "sometidos a cierta preparación y formados después como bollos, como unos queques de chocolate" (Witt, 1987: 91).

Otros varios términos proceden de esa época, algunos con vida efímera. Hacia 1840, Felipe Pardo y Aliaga ponía ya de manifiesto nítidamente, en El espejo de mi tierra, la ridícula y ciega admiración a los extranjeros, que sufrían muchos limeños, con lo que el afán por emplear vocablos nuevos y extraños no es una actitud exclusiva del siglo XX:

Fulano va muy orondo, enganchado del brazo de un extranjero, a quien pasea por todas partes, a quien presenta en las casas principales, a quien luce en todos los espectáculos (...). ¿Es algún príncipe, algún noble que viaja por economía, algún sabio que quiere enriquecer la literatura con el fruto de sus peregrinaciones, algún artista, algún negociante de esmerada educación? No, señor, nada de eso: no es más que un contramaestre. Aquella remilgada damisela no sabe palabra de inglés, ni de francés, ni de italiano, ni de alemán (se entiende, dejándole a salvo su derecho a hablar mal el castellano); pero siempre ha de estar circundada de extranjeros. (1971:78)

En realidad, no solo vinieron contramaestres con los vapores que hacían la ruta de Valparaíso a San Francisco. Muchos viajeros anglosajones se interesaron por los países andinos. $\mathrm{Si}$ exceptuamos a los piratas y corsarios cuya huella principal fue la destrucción y el saqueo, y militares que intervinieron en las guerras de Independencia como Cochrane o Miller, tal vez el primer viajero que llegó a estas costas pudo ser David Porter, marino norteamericano que recogió impresiones sobre Chile, Perú y las Galápagos entre 1812 y 1814. También hay que considerar las noticias de Anthony Helms y Samuel Haigh, pero tal vez el más conocido y ameno relato fue el de Basilio Hall, que presenció la declaración del 28 de julio de 1821 . También destaca el famoso novelista Robert Louis Stevenson, que presenció en Lima el consiguiente saqueo del tribunal de Inquisición.

Otro viajero importante fue Henry Lister Maw, autor de Journal of a passage from the Pacific to the Atlantic (Londres, 1829). Su viaje se inició en Lima y llegó al Amazonas pasando de Cajamarca a Chachapoyas y Moyobamba. Por su parte, el primero que plasmó sus impresiones en la prensa norteamericana fue el caricaturista satírico G. W. Carleton, que ofreció una visión descarnada y despreciativa del Perú.

James Orton elaboró un estudio etnográfico: The Andes and the Amazon across the Continent of South America (Nueva York, 1876). Richard Spruce llegó en 1863 para indagar las posibles ventajas del cultivo de algodón en Perú: Notes on the valleys of Piura and Chira (Londres, 1864). El mundo andino se convertía en una extensión de los estudios americanos y el espejo donde proyectar sus quejas y aspiraciones. De ese modo, también Perú se empezó también a conocer en los Estados Unidos, también a través de las recetas milagrosas del naturalista Paul Fontain, quien difundiría en Estados Unidos el poder de la cocaína, "the most powerful and permanent stimulant known", en un estudio más tardío: The great mountains and forests of South America (1904). 
Los préstamos lingüísticos suelen tener direcciones recurrentes, pero también las oleadas de anglicismos tuvieron un reflujo en palabras que del español americano pasaron al inglés británico y norteamericano. Y así, el inglés adaptó palabras del quechua como guano, cóndor, llama y pampa. Los viajeros ponen en cursiva palabras como tambo o locro. El inglés americano incorporó "charqui", convertido en "jerky", común en todo Sudoeste de Estados Unidos. El peruanismo "pallar" se conoce en Estados Unidos como "lima bean", o sea, traducido literalmente: "frijol de Lima".

El primer repertorio lexicográfico que registra "faite" es el del padre Rubén Vargas Ugarte, describiéndolo con sinónimos: "matón, valiente, guapo" (1953: 21). Era término de germanía, o sea jerga de delincuentes. Pero en un relato de Raúl Estuardo Cornejo aparece un bandolero que enamoraba a todas las mujeres: "Como que se la daba de faite, según el decir" (2007: 154). Ha cambiado su significado.

Según José Gálvez, fue en la época de la guerra con Chile cuando aparece el término faite y faiteman en las calles de Lima, que tuvieron hasta indumentaria propia: "Abusivos y fachendosos, complacáinse en golpear a cuanto desgraciado se ponía a sus alcances", y había incluso niños que por el afán de parecerse a los faites hablaban "imitando el tono faitoso" (Ś1921Ṣ1947: 55-57).

Parece que no ha mantenido vitalidad suficiente el adjetivo "esplinático", con que se autodenominaba José Carlos Mariátegui (decía ser "poeta esplinático"), en clara alusión al spleen de Charles Baudelaire. El término se había puesto de moda entre los intelectuales en la España de Bretón de los Herreros, pero en el siglo XX todavía la utiliza Enrique López Albujar en su composición Anoche estuve en Piura (1951): "para así convencerme / de que me hallaba dentro de la realidad / y luego de lanzar un esplinático bostezo" (1954: XVII).

$Y$ es interesante que los anglicismos hayan creado fraseología en español. Pasa con la frase "poner al breque" que vale por 'detener', 'frenar', 'apaciguar', y también 'enseñar'. Procede de la época de los primeros ferrocarriles, de donde vino "breque", 'freno' (del inglés "brake"), y se derivó "brequero", para referirse al guardafrenos. Es usual en Perú y Ecuador. La locución cuenta con un registro lexicográfico temprano en los Barbarismos fonéticos del Ecuador de Gustavo Lemos Ramírez: "Al desenfrenado que se le detiene con castigos correccionales o amenazas se le pone al breque." (1922: 23). Martha Hildebrandt (El Comercio, 15/4/2015) señala también otra frase del mismo origen: "meter en breque" para decir castigar o educar especialmente a los niños. Pero "al breque" ha tenido mayor difusión y lo muestra este ejemplo de Julio Ramón Ribeyro: "Ya Alida piensa en el remedio tradicional: un buen internado, donde lo tengan al breque" (1995: 217).

Por otra parte, en Perú, México, El Salvador, Costa Rica, Guatemala, Cuba y Colombia, se dice "la del estribo" a la copa o el trago que se toma uno al final de una reunión o, simplemente, al despedirse. Un ejemplo de Bryce Echenique: "Y también, humano muy humano, aceptó la secreta invitación que le hizo Nadine para tomarse la del estribo en su cuarto, escuchando un poco de música, conversando un rato, y mirando la luna llena de lluvia por la claraboya" (1981: 166).

También aparece en este pasaje de Mario Vargas Llosa: "La Selvática y el Bolas lo trajeron hasta la mesa, y Josefino no estaba en las últimas, qué cojudeces, la del estribo era de él, que nadie se mueva, y que la Chinguita se bajara una cervecita" (1967: 373). En México se refiere también a la última canción y en Argentina aplican la expresión al mate "del estribo" con el mismo sentido, pues es el que toma uno antes de irse de la ronda.

La frase es un calco que se explica por esta temprana influencia del inglés en el español de los países suramericanos. Lo que la hace tal vez un poco más interesante es que fue tomada del inglés cuando en ese idioma estaba casi en desuso y conforme se ha extendido en español americano ha ido desapareciendo del inglés estadounidense, que la reemplazó por otra frase: "one for the road". Debió haberse trasmitido al español americano por alguno de esos viajeros ingleses en el siglo XIX, antes 
de desaparecer en el primer tercio del siglo $\mathrm{XX}$. Uno de ellos fue el británico Samuel Haigh, que ya mencioné. Cuando Alberto Tauro del Pino traduce las descripciones del viajero, no tiene ninguna dificultad para usar la expresión.

Una docena de amigos me acompañaron hasta Uchumayo donde bebimos la copa del estribo y, en compañía de nuestro cónsul, míster Passmore, que se trasladaba al puerto por negocios, me despedí de Arequipa. (Haigh, 1967: 29).

El idioma guarda testimonios de la historia recorrida por sus hablantes $y$ en este fluir constante hay afluentes que proceden del contacto con viajeros, comerciantes y aventureros anglosajones. Ahora, son parte de nuestro castellano.

\section{REFERENCIAS}

Alfaro, Ricardo Joaquín, (1970) Diccionario de anglicismos, Madrid, Gredos.

Arona, Juan de, (seudónimo de Pedro Paz Soldán y Unanue) (1974) Diccionario de peruanismos, Edición de Estuardo Núñez, Lima, Peisa, 2 vols.

Arrizabalaga, Carlos, (2003) "Barbarismos en las Papeletas lexicográficas de Ricardo Palma", en Revista de la Casa Museo Ricardo Palma, 4, p. 13-28.

Bryce Echenique, Alfredo, (1981) La vida exagerada de Martín Romaña. Barcelona, Anagrama. Casares, Julio, (1950) Introducción a la lexicografía moderna. Madrid, CSIC.

Cornejo, Raúl Estuardo, (2007) Horizontes de sol. Cuentos tempranos. Lima, Editorial San Marcos.

Corrales, Juan Apapucio [seudónimo de Clemente Palma] (1938) Crónicas político-domésticotaurinas, Lima, Compañía de Impresiones y Publicidad.

Foley Gambetta, Enrique, (1983) Léxico del Perú: peruanismos, replana criolla, jerga del hampa, regionalismos, provincialismos, locuciones, modismos, etc. usuales en el Perú. Lima.

Gálvez, José, (1947) Una Lima que se va. Lima, Editorial PTCM.

Haigh, Samuel, (1967) "Bosquejos del Perú. 1825-1827”, en Alberto Tauro del Pino, Viajeros en el Perú republicano. Lima, Universidad Nacional Mayor de San Marcos.

Hildebrandt, Martha, (1994) Peruanismos. Lima, Biblioteca Nacional del Perú.

Lapesa, Rafael, (1996) El español moderno y contemporáneo. Estudios lingüísticos. Barcelona, Crítica.

Mallo, Jerónimo, (1959) "La invasión del anglicismo en la lengua española de América", Cuadernos Americanos, XVIII (4), pp. 115-123.

Palma, Ricardo, (2003) Papeletas lexicográficas. Dos mil setecientas voces que hacen falta en el Diccionario. Lima, Imprenta La Industria, 1903. Nueva edición en Lima, Academia Peruana de la Lengua. 
Palma, Ricardo, (1964). Tradiciones peruanas completas. Madrid, Aguilar, 1964.

Pardo y Aliaga, Felipe, (1971) El espejo de mi tierra. Edición de Alberto Tauro. Lima, Universo.

Ribeyro, Julio Ramón, (1995) La tentación del fracaso. Memorias. Lima, Campodónico.

Seco, Manuel, (1993) "La otra voz de la Academia Española. Notas sobre el Diccionario Manual", en David Mackenzie y Ian Michael, Hispanic Linguistic Studies in Honour of F. W. Hodcroft, Llangrannog (Wales): The Dolphin Book, pp. 153-196.

Segura, Manuel Ascensio, (1968) Artículos de costumbres, Lima, Universo.

Vargas Llosa, Mario, (1967) La Casa Verde, Barcelona, Seix Barral.

Vargas Ugarte, Rubén, (1953) Glosario de peruanismos. Lima.

Witt, Heinrich, Diario y observaciones sobre el Perú (1824-1890) (1987) Selección de Pablo Macera. Lima, COFIDE.

Fecha de recepción: 26-01-16

Fecha de aceptación: 02-06-16 